\title{
Effect of nutritional supplementation with milk whey proteins in amyotrophic lateral sclerosis patients
}

\author{
Luciano Bruno de Carvalho Silva', Lucia Figueiredo Mourão², \\ Ariovaldo Armando Silva3 ${ }^{3}$ Núbia Maria Freire Vieira Lima ${ }^{4}$, \\ Sara Regina Almeida ${ }^{4}$, Marcondes C. Franca Jr ${ }^{5}$, \\ Anamarli Nucci ${ }^{6}$, Jaime Amaya-Farfán ${ }^{7}$
}

\begin{abstract}
Objective: We evaluated the efficacy of oral supplementation with milk whey proteins and modified starch (70\%WPI:30\%MS), on nutritional and functional parameters of patients with ALS. Method: A prospective randomized double-blind study was performed with 16 ALS patients, divided in two groups, the treatment group received (70\%WPI:30\%MS) and the control group received (maltodextrin). They underwent prospective nutritional and functional assessment for 4 months. Results: Patients in the treatment group presented weight gain, increased body mass index (BMI), increased arm muscle area and circumference, higher albumin, white blood cell and total lymphocyte counts, and reduced creatine-kinase, aspartate aminotransferase and alanine aminotransferase. In the control group, biochemical parameters did not change, but weight and BMI declined. Conclusion: Our results indicate that the agglomerate $70 \% \mathrm{WPI}: 30 \% \mathrm{MS}$ may be useful in the nutritional therapy of patients with ALS.
\end{abstract}

Key words: amyotrophic lateral sclerosis, nutrition status, anthropometry.

\section{Efeito da suplementação nutricional com proteínas do soro de leite em pacientes com esclerose lateral amiotrófica}

\section{RESUMO}

Objetivo: Avaliar a eficácia da suplementação nutricional oral com proteínas do soro do leite e amido modificado (70\%WPI:30\%MS), nos parâmetros nutricionais e funcionais de pacientes com esclerose lateral amiotrófica (ELA). Método: Foi realizado estudo randomizado duplo-cego, com 16 pacientes com ELA, divididos em dois grupos, um que recebeu 70\%WPI:30\%MS e um controle que recebeu maltodextrina. Os pacientes foram submetidos a avaliação nutricional e funcional durante quatro meses. Resultados:

Correspondence

Gabriel Monteiro da Silva Departamento Nutricional Universidade Federal de Alfenas 37130-000 Alfenas MG - Brasil

E-mail: luciano@unifal-mg.edu.br

Financial support

CNPq - Conselho Nacional

de Desenvolvimento

Científico e Tecnológico

Received 24 September 2009

Received in final form 12 November 2009 Accepted 18 November 2009
Nos pacientes que receberam o suplemento $70 \% \mathrm{WPI}: 30 \% \mathrm{MS}$, foi observado ganho de peso, aumento na contagem de linfócitos e redução de creatina kinase, aspartato aminotransferase and alanina aminotransferase. No grupo controle, os parâmetros bioquímicos não sofreram modificações; no entanto, peso e índice de massa corporal diminuíram. Conclusão: Nossos resultados indicam que o aglomerado 70\%WPI:30\%MS pode ser útil na terapia de pacientes com ALS.

Palavras-chave: esclerose lateral amiotrófica, estado nutricional, antropometria.

${ }^{1} M D$, Prof., Federal University of Alfenas- MG (UNIFAL-MG) and Center for Food Security Studies (NEPA), University of Campinas (UNICAMP), Campinas SP, Brazil; ${ }^{2}$ MD, Prof. PhD, Speech Pathologist Medical School (FCM/UNICAMP); ${ }^{3}$ MD, Prof.,

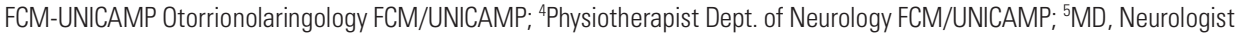
Ambulatory Neuromuscular Diseases HC/UNICAMP; ${ }^{6}$ MD, Associate Prof., FCM/UNICAMP. Ambulatory Neuromuscular Diseases HC/UNICAMP; ${ }^{7} \mathrm{MD}$, Full Prof. FEA/UNICAMP, NEPA/UNICAMP. 
Amyotrophic lateral sclerosis (ALS) is a progressive neurodegenerative disorder of motor neurons in the cerebral cortex, brainstem, and spinal cord ${ }^{1}$. ALS patients show multiple clinical features, since the disease affects bulbar and/or spinal systems according to the site of motor neuron involvement. As the disease progresses, both upper and lower motor neurons may be affected, and various symptoms may overlap ${ }^{2}$. During the course of ALS, there is often a decline in nutritional status that is not properly managed in clinical practice, even though it is a significant and independent prognostic survival factor ${ }^{3}$.

Nutritional status should be routinely monitored to identify poor food intake and malnutrition ${ }^{4}$. Patients may experience rapid weight loss associated with reduced nutritional intake, prolonged meal times, fatigue, dehydration and depression. Unfortified texture-modified diets for dysphagic patients may hasten weight loss due to their low protein and energy content $t^{5,6}$. When these nutritiondepleting co-factors are present the insidious onset of malnutrition is inevitable.

Ingredients such as milk whey proteins, when chemically or physically modified, can alter the viscosity of food systems. However, on their own, they are unable to reach the viscosity standardised by the $\mathrm{ADA}^{7}$ for fluids, but agglomerated with carbohydrate sources such as modified starch, they could increase the viscosity of food systems to values close to those cited by the ADA.

Based on the high malnutrition index and difficulty in nutritional handling, the main objective of this study was to evaluate the effect of supplementation with the agglomerate with $70 \%$ milk whey protein isolate and 30\% modified starch (70\%WPI:30\%MS), on anthropometrics, body composition and biochemists parameters in ALS patients.

\section{METHOD}

\section{Study design and population}

We performed a prospective randomized doubleblind controlled clinical trial to evaluate oral supplementation of milk whey protein in patients with ALS. We recruited 16 patients (14 males) with clinically definite ALS from the Neuromuscular outpatient clinic of Campinas University (UNICAMP). Patients were divided in two groups of 8 patients each, and randomized to receive either milk whey protein or maltodextrin (control), for 16 weeks, and submitted to bimonthly evaluation.

The study was conducted between July 2006 and July 2007. The study was approved by the ethics committee of the School of Medical Sciences - UNICAMP and all patients accepted a written consent.

\section{Inclusion criteria}

ALS patients meeting El Escorial criteria $(1998)^{8}$ for defined disease, either with bulbar or appendicular onset, regularly assisted in the Clinic, were included in the study.

\section{Exclusion criteria}

Patients with nasogastric tube or gastrostomy, on assisted mechanical ventilation and without intervening neurological illnesses.

\section{Nutritional intervention phase}

Patients were randomized to receive either maltodextrin (control group) or oral supplementation with whey protein (treatment group). This is a nutritional supplement developed at UNICAMP containing 70\% of milk serum protein and 30\% modified starch (70\% WPI:30\% MS). Patients received maltodextrin or whey protein for 16 weeks. Nutritional assessment, including anthropometric and biochemical measures, was performed at the onset, two months and four months after supplementation. We performed functional assessment at the onset and four months after supplementation.

\section{Nutritional supplementation}

Individual daily needs of protein are $1.2 \mathrm{~g} / \mathrm{kg}$ of body weight. We offered whey protein to the patients, approximately $30 \%$ of the daily protein requirements.

Nutritional supplementation was administered twice a day (morning and afternoon) for 16 consecutive weeks. Patients did not receive the supplement concurrently with Riluzole. They were instructed to keep the product in a refrigerator and to solubilize it before administration; on an individual basis, assays to the adequate viscosity were performed with orange juice ( $\mathrm{pH}=4.0$ and $1.5 \%$ of solid components).

\section{Dietary evaluation}

After nutritional anamnesis and socio-economic data collection, we applied the $24 \mathrm{~h}$ record method ${ }^{9}$ in 6 different days for every patient. We inquired about the usual diet of the patient, including amount, commercial brand and the cooking procedure. We estimated energy requirement (ER); proportion of carbohydrates, proteins and lipids; proportion of saturated, polyunsaturated and monounsaturated fat relative to ER; and the intake of fibers, calcium, iron, retinol and ascorbic acid. Estimates were accomplished by the software DIET PRO $4.0^{10}$. The amount of fibers, cholesterol, saturated, polyunsaturated and monounsaturated fat was analyzed by DIET PRO 4.0 in accordance with the Brazilian food composition table ${ }^{11}$ and the table of the United States Department of Agriculture (USDA - Release 16) ${ }^{12}$.

We evaluated macronutrient and protein nutritional adequacy in accordance with the Recommended Di- 
etary Allowances (RDA) ${ }^{13}$ and the recommendations of the Brazilian Society of Food and Nutrition ${ }^{14}$, respectively. We used the guidelines of the Institute of Medicine to analyze intake of micronutrients. The Harris and Benedict equation, modified by Long et al. ${ }^{15}$ was employed to analyze resting energy requirements.

\section{Nutritional evaluation}

Body weight $(\mathrm{kg})^{16}$ and height $(\mathrm{m})$ were assessed, owing to the fact that four patients older than 60 were limited to wheelchairs, the Chumlea et al. method was used ${ }^{17}$. Body mass index (BMI $-\mathrm{kg} / \mathrm{m}^{2}$ ) and midarm circumference (MAC - $\mathrm{cm})^{18,19}$ were determined; the tricipital (TSF), bicipital (BSF), supra-iliac (SISF) and subscapular (SESF) skinfolds (SF) were measured using the scientific Lange branded $0.1 \mathrm{~mm}$-accuracy adipometer, in order to classify ALS patients according to the estimates of relative body fat ${ }^{18}$; midarm muscle circumference (MAMC), arm muscle area (AMA) and arm fat area (AFA) were obtained from MAC and TSF according to Heymsfield et al. and Frisancho ${ }^{19,20}$. The percentage of weight loss (\%WL) was determined based upon the usual and the actually measured weight of the patient ${ }^{21}$. The protein-caloric malnutrition score (PCMS $)^{18}$ was used to classify nutritional status of patients.

\section{Clinical and biochemical parameters}

We determined the serum levels of $\mathrm{C}$-reactive protein, albumin, pre-albumin, creatine-kinase (CK), creatine, urea, glucose, aspartate transferase (AST), alanine transferase (ALT), total lymphocyte count, platelets, sodium and potassium for each patient.

Table 1. Influence of the nutritional supplementation with the 70\%WPI:30\%MS agglomerate on body weight (kg), body mass index (BMI$\mathrm{kg} / \mathrm{m}^{2}$ ), fat mass (\%) and fat free mass (\%), tricipital skinfold (TSF), bicipital skinfold (BSF), supra-iliac skinfold (SISF), subscapular skinfold (SESF) and sum of the four skinfolds (mm), midarm circumference (MAC), wrist circumference, midarm muscle circumference (MAMC), arm muscle area (AMA) and arm fat area (AFA) $\left(\mathrm{cm}^{2}\right)$.

\begin{tabular}{|c|c|c|c|c|c|c|}
\hline \multirow[b]{3}{*}{ Parameters } & \multicolumn{3}{|c|}{ Control group* } & \multicolumn{3}{|c|}{ Treatment group* } \\
\hline & \multicolumn{3}{|c|}{ Months } & \multicolumn{3}{|c|}{ Months } \\
\hline & 0 & 2 & 4 & 0 & 2 & 4 \\
\hline Body weight (kg) & $\begin{array}{l}65.8 \pm 1.2^{\mathrm{a}} \\
{[49.8-81.0]}\end{array}$ & $\begin{array}{l}64.7 \pm 1.3^{b} \\
{[48.0-80.0]}\end{array}$ & $\begin{array}{l}64.3 \pm 1.3^{b} \\
{[48.0-80.0]}\end{array}$ & $\begin{array}{c}62.3 \pm 1.5 \\
{[45.4-81.5]}\end{array}$ & $\begin{array}{c}63.3 \pm 1.5 \\
{[46.1-81.6]}\end{array}$ & $\begin{array}{c}63.4 \pm 1.5 \\
{[45.9-81.7]}\end{array}$ \\
\hline BMI $\left(k g / m^{2}\right)$ & $\begin{array}{l}22.9 \pm 0.4^{\mathrm{a}} \\
{[17.2-26.9]}\end{array}$ & $\begin{array}{l}22.5 \pm 0.4^{b} \\
{[17.0-27.7]}\end{array}$ & $\begin{array}{l}22.2 \pm 0.4^{b} \\
{[17.0-27.7]}\end{array}$ & $\begin{array}{c}21.7 \pm 0.4^{B} \\
{[18.12-27.03]}\end{array}$ & $\begin{array}{c}22.1 \pm 0.4^{\mathrm{A}} \\
{[18.20-27.17]}\end{array}$ & $\begin{array}{c}22.3 \pm 0.5^{\mathrm{A}} \\
{[18.43-27.51]}\end{array}$ \\
\hline Fat mass (\%) & $\begin{array}{c}26.1 \pm 0.7 \\
{[15.6-40.4]}\end{array}$ & $\begin{array}{c}28.1 \pm 0.3 \\
{[15.6-39.9]}\end{array}$ & $\begin{array}{c}27.3 \pm 0.3 \\
{[15.6-40.0]}\end{array}$ & $\begin{array}{c}20.8 \pm 0.6 \\
{[10.38-26.5]}\end{array}$ & $\begin{array}{c}20.5 \pm 0.6 \\
{[10.2-25.5]}\end{array}$ & $\begin{array}{c}20.4 \pm 0.6 \\
{[10.01-25.9]}\end{array}$ \\
\hline Fat free mass (\%) & $\begin{array}{c}73.8 \pm 0.3 \\
{[59.6-84.4]}\end{array}$ & $\begin{array}{c}71.9 \pm 1.2 \\
{[60.1-84.4]}\end{array}$ & $\begin{array}{c}72.7 \pm 1.2 \\
{[60.0-84.4]}\end{array}$ & $\begin{array}{c}79.1 \pm 0.6 \\
{[73.5-89.62]}\end{array}$ & $\begin{array}{c}79.5 \pm 0.6 \\
{[74.5-89.8]}\end{array}$ & $\begin{array}{c}79.6 \pm 0.6 \\
{[74.189 .99]}\end{array}$ \\
\hline TSF $(\mathrm{mm})$ & $\begin{array}{c}14.9 \pm 0.5 \\
{[7-23]}\end{array}$ & $\begin{array}{c}14.4 \pm 0.5 \\
{[7-23]}\end{array}$ & $\begin{array}{c}15.8 \pm 0.5 \\
{[6.5-24]}\end{array}$ & $\begin{array}{c}9.6 \pm 0.2^{B} \\
{[6-12]}\end{array}$ & $\begin{array}{c}10.7 \pm 0.2^{\mathrm{A}} \\
{[7-13]}\end{array}$ & $\begin{array}{c}11.1 \pm 1.2^{\mathrm{A}} \\
{[8-13]}\end{array}$ \\
\hline $\mathrm{BSF}(\mathrm{mm})$ & $\begin{array}{c}7.4 \pm 0.3^{b} \\
{[3-12]}\end{array}$ & $\begin{array}{c}7.4 \pm 0.2^{\mathrm{ab}} \\
{[4-12]}\end{array}$ & $\begin{array}{l}8.6 \pm 0.2^{\mathrm{a}} \\
{[3.5-12.6]}\end{array}$ & $\begin{array}{c}6.2 \pm 0.1^{\mathrm{A}} \\
{[4-8]}\end{array}$ & $\begin{array}{c}5.6 \pm 0.1^{\mathrm{B}} \\
{[4-7]}\end{array}$ & $\begin{array}{c}5.5 \pm 0.1^{\mathrm{B}} \\
{[4-7.1]}\end{array}$ \\
\hline SISF (mm) & $\begin{array}{c}15.5 \pm 0.7 \\
{[5-25]}\end{array}$ & $\begin{array}{c}16.1 \pm 0.7 \\
{[5-25]}\end{array}$ & $\begin{array}{c}16.2 \pm 0.7 \\
{[5-26]}\end{array}$ & $\begin{array}{c}12.4 \pm 0.3 \\
{[6-18]}\end{array}$ & $\begin{array}{c}11.8 \pm 0.3 \\
{[6-17]}\end{array}$ & $\begin{array}{c}11.7 \pm 0.3 \\
{[6-17]}\end{array}$ \\
\hline SESF (mm) & $\begin{array}{c}12.9 \pm 0.4^{b} \\
{[8-22]}\end{array}$ & $\begin{array}{c}13.5 \pm 0.5^{\mathrm{a}} \\
{[8-22.3]}\end{array}$ & $\begin{array}{c}13.6 \pm 0.5^{\mathrm{a}} \\
{[8-22.4]}\end{array}$ & $\begin{array}{c}11.5 \pm 0.2^{\mathrm{A}} \\
{[8-14]}\end{array}$ & $\begin{array}{c}10.8 \pm 1.2^{B} \\
{[8-13.8]}\end{array}$ & $\begin{array}{c}10.2 \pm 0.2 C \\
{[8-13.5]}\end{array}$ \\
\hline$\Sigma \mathrm{SF}(\mathrm{mm})$ & $\begin{array}{c}50.6 \pm 1.5 \\
{[24-71]}\end{array}$ & $\begin{array}{c}51.4 \pm 1.5 \\
{[25-71]}\end{array}$ & $\begin{array}{l}66.6 \pm 1.6 \\
{[23.1-3.8]}\end{array}$ & $\begin{array}{c}39.8 \pm 0.4 \\
{[35-46]}\end{array}$ & $\begin{array}{l}39.1 \pm 0.3 \\
{[34.8-44]}\end{array}$ & $\begin{array}{l}38.5 \pm 0.3 \\
{[34.5-43]}\end{array}$ \\
\hline $\mathrm{MAC}(\mathrm{cm})$ & $\begin{array}{l}27.2 \pm 0.3 \\
{[20.5-31]}\end{array}$ & $\begin{array}{c}27.3 \pm 0.1 \\
{[20-31]}\end{array}$ & $\begin{array}{c}27.2 \pm 0.1 \\
{[20-31]}\end{array}$ & $\begin{array}{c}25.3 \pm 0.5 \\
{[19.5-30.8]}\end{array}$ & $\begin{array}{l}25.5 \pm 0.5 \\
{[19.5-30]}\end{array}$ & $\begin{array}{c}25.8 \pm 0.4 \\
{[21-30]}\end{array}$ \\
\hline wrist circ (cm) & $\begin{array}{l}16.7 \pm 0.1 \\
{[14.4-18]}\end{array}$ & $\begin{array}{c}17.1 \pm 0.1 \\
{[15-18]}\end{array}$ & $\begin{array}{c}16.7 \pm 0.1 \\
{[14.5-18.2]}\end{array}$ & $\begin{array}{l}17.1 \pm 0.2 \\
{[14.8-19]}\end{array}$ & $\begin{array}{l}17.1 \pm 1.3 \\
{[14.8-19]}\end{array}$ & $\begin{array}{l}17.1 \pm 1.3 \\
{[14.8-19]}\end{array}$ \\
\hline MAMC (cm) & $\begin{array}{l}23.3^{\mathrm{a}} \pm 0.3 \\
{[18.3-26.5]}\end{array}$ & $\begin{array}{c}22.8 \pm 0.3 \\
{[17.8-26.9]}\end{array}$ & $\begin{array}{l}22.6 \pm 0.3^{b} \\
{[17.9-27.1]}\end{array}$ & $\begin{array}{l}22.5 \pm 0.5 \\
{[15.4-27.3]}\end{array}$ & $\begin{array}{c}22.8 \pm 0.5 \\
{[15.7-28.3]}\end{array}$ & $\begin{array}{c}23.9 \pm 0.5 \\
{[16.9-28.4]}\end{array}$ \\
\hline AMA $\left(\mathrm{cm}^{2}\right)$ & $\begin{array}{l}42.1 \pm 2.1^{a} \\
{[15.9-67.8]}\end{array}$ & $\begin{array}{l}42.1 \pm 2.1^{a} \\
{[15.9-67.2]}\end{array}$ & $\begin{array}{c}39.9 \pm 2.1^{b} \\
{[14.4-67]}\end{array}$ & $\begin{array}{c}47.8 \pm 2.5 \\
{[23.1-69.9]}\end{array}$ & $\begin{array}{c}48.6 \pm 2.3 \\
{[30.1-71.9]}\end{array}$ & $\begin{array}{c}48.4 \pm 2.2 \\
{[31.0-71.6]}\end{array}$ \\
\hline AFA $\left(\mathrm{cm}^{2}\right)$ & $\begin{array}{l}61.3 \pm 1.4^{\mathrm{a}} \\
{[33.475 .4]}\end{array}$ & $\begin{array}{l}61.4 \pm 1.4^{a} \\
{[33.9-76.4]}\end{array}$ & $\begin{array}{l}64.2 \pm 1.4^{b} \\
{[34-78.2]}\end{array}$ & $\begin{array}{l}20.9 \pm 0.6^{A} \\
{[10.3-26.5]}\end{array}$ & $\begin{array}{l}20.1 \pm 0.6^{B} \\
{[10.2-25.5]}\end{array}$ & $\begin{array}{l}19.8 \pm 0.6^{B} \\
10.1-25.2]\end{array}$ \\
\hline
\end{tabular}

*Values correspond to means \pm standard error of three determinations and minimum and maximum values. Values not sharing similar letter in the same line referent to control group (a) and treatment group (A) are different $(p<0.05)$ in Tukey test. 


\section{Amyotrophic Lateral Sclerosis Functional Rating Scale Revised (ALSFRS-R)}

ALSFRS- $R$ is a questionnaire-based scale for activities of daily living. This scale contains 12 items grouped into three domains that encompass appendicular function (gross motor tasks), bulbar and respiratory function. Each item has a 5 -point scale ( 0 for unable. 4 for normal) and scores ranging from 0 to 48 . Low scores denote a serious disease status ${ }^{22}$.

The patients were evaluated by an interdisciplinary group. The nutritional assessment was done by a nutritionist and the ALSFRS-R assessment by physiotherapists.

\section{Statistical analysis}

We used the Kolmogorov-Smirnov test to evaluate whether the studied variables had normal distribution. Independent variables with normal distribution were expressed as mean \pm standard deviation, and compared with a two-sided t test. Prospective data were analyzed with a paired t-test. We used the Mann-Whitney test to compare independent groups without normal distribution. The value was set at $5 \%$.

\section{RESULTS}

The average age of the patients was 53 (ranging between 32 and 69 years old) and the illness time, 2 years \pm 1 . In 10 patients, the impairment was predominantly appendicular, whereas in the remaining 6 it was bulbar (GB).

We found increased food intake in both control and supplemented groups after nutritional intervention. In both groups, patients followed nutritional advice as shown by the relevant improvement in macro and micronutrient intake.

At the time of enrollment, patients in the control group exhibited $77 \%$ nutritional adequacy, but after 4 months, the percentage rose to $90.27(\mathrm{p}=0.032)$. Carbohydrate intake $(70.8 \%$ vs $82 \%, \mathrm{p}=0.022)$ significantly increased in 4 months, as did the lipid intake (93\% vs

Table 2. Influence of nutritional supplementation with the 70\%WPI:30\%MS agglomerate on clinical and biochemical parameters.

\begin{tabular}{|c|c|c|c|c|c|c|}
\hline \multirow[b]{3}{*}{ Parameters } & \multicolumn{3}{|c|}{ Control group* } & \multicolumn{3}{|c|}{ Treatment group* } \\
\hline & \multicolumn{3}{|c|}{ Months } & \multicolumn{3}{|c|}{ Months } \\
\hline & 0 & 2 & 4 & 0 & 2 & 4 \\
\hline C-reative protein (mg/dL) & $\begin{array}{c}0.21 \pm 0.03 \\
{[0.04-0.7]}\end{array}$ & $\begin{array}{l}0.21 \pm 0.03 \\
{[0.05-0.74]}\end{array}$ & $\begin{array}{c}0.18 \pm 0.02 \\
{[0.05-0.5]}\end{array}$ & $\begin{array}{l}0.10 \pm 001^{B} \\
{[0.02-0.23]}\end{array}$ & $\begin{array}{c}0.13 \pm 0.01^{\mathrm{A}} \\
{[0.02-0.28]}\end{array}$ & $\begin{array}{l}0.12 \pm 0.03 \\
{[0.02-0.27]}\end{array}$ \\
\hline Albumin (g/dL) & $\begin{array}{c}4.51 \pm 0.03 \\
{[4.15-4.9]}\end{array}$ & $\begin{array}{c}4.47 \pm 0.04 \\
{[4.0-5.1]}\end{array}$ & $\begin{array}{c}4.48 \pm 0.05 \\
{[3.9-5.3]}\end{array}$ & $\begin{array}{c}4.30 \pm 0.05^{c} \\
{[3.6-4.8]}\end{array}$ & $\begin{array}{c}4.51 \pm 0.03^{B} \\
{[4.2-4.9]}\end{array}$ & $\begin{array}{c}4.76 \pm 0.01^{\mathrm{A}} \\
{[4.4-4.9]}\end{array}$ \\
\hline Pre-Albumin (mg/dL) & $\begin{array}{c}28.2 \pm 0.7 \\
{[19.5-39.1]}\end{array}$ & $\begin{array}{c}29 \pm 0.8 \\
{[17.2-34.3]}\end{array}$ & $\begin{array}{c}29.2 \pm 0.9 \\
{[19.5-36.7]}\end{array}$ & $\begin{array}{c}26.3 \pm 0.6 \\
{[17.7-32.3]}\end{array}$ & $\begin{array}{c}30.6 \pm 0.5 \\
{[35.4-23.6]}\end{array}$ & $\begin{array}{c}30.6 \pm 0.7 \\
{[24.5-39.2]}\end{array}$ \\
\hline CK (U/L) & $\begin{array}{c}338.7 \pm 31.1 \\
{[100-774]}\end{array}$ & $\begin{array}{l}310 \pm 27 \\
{[87-645]}\end{array}$ & $\begin{array}{c}335.5 \pm 29.4 \\
{[84-626]}\end{array}$ & $\begin{array}{c}449.6 \pm 30.3^{\mathrm{A}} \\
{[117-795]}\end{array}$ & $\begin{array}{c}375 \pm 31.6^{\mathrm{AB}} \\
{[87-888]}\end{array}$ & $\begin{array}{c}307 \pm 12.2^{B} \\
{[143-403]}\end{array}$ \\
\hline Urea (mg/dL) & $\begin{array}{c}29.5 \pm 0.8 \\
{[22-43]}\end{array}$ & $\begin{array}{c}29.25 \pm 0.9 \\
{[20-45]}\end{array}$ & $\begin{array}{c}29.5 \pm 1.2 \\
{[17-40]}\end{array}$ & $\begin{array}{c}27.5 \pm 1.1^{\mathrm{A}} \\
{[19.9-46]}\end{array}$ & $\begin{array}{c}26.1 \pm 0.7^{B} \\
{[22-43]}\end{array}$ & $\begin{array}{c}26.7 \pm 0.8 \\
{[19-42]}\end{array}$ \\
\hline Creatine $(\mathrm{mg} / \mathrm{dL})$ & $\begin{array}{l}0.56 \pm 0.02 \\
{[0.39-1.02]}\end{array}$ & $\begin{array}{c}0.63 \pm 0.03 \\
{[0.4-1.06]}\end{array}$ & $\begin{array}{l}0.57 \pm 0.03 \\
{[0.35-1.13]}\end{array}$ & $\begin{array}{l}0.74 \pm 0.02 \\
{[0.35-1.02]}\end{array}$ & $\begin{array}{c}0.63 \pm 0.03 \\
{[0.25-1.0]}\end{array}$ & $\begin{array}{c}0.61 \pm 0.03 \\
{[0.25-1.1]}\end{array}$ \\
\hline Glucose (mg/dL) & $\begin{array}{l}85 \pm 1.7 \\
{[55-99]}\end{array}$ & $\begin{array}{l}86 \pm 1.5 \\
{[72-103]}\end{array}$ & $\begin{array}{l}89 \pm 1.4 \\
{[76-111]}\end{array}$ & $\begin{array}{c}81.6 \pm 0.7 \\
{[74-90]}\end{array}$ & $\begin{array}{l}80 \pm 0.7 \\
{[73-88]}\end{array}$ & $\begin{array}{l}80 \pm 0.8 \\
{[69-87]}\end{array}$ \\
\hline AST (U/L) & $\begin{array}{c}27.1 \pm 1.1 \\
{[13-41]}\end{array}$ & $\begin{array}{c}25.6 \pm 0.8 \\
{[14-34]}\end{array}$ & $\begin{array}{c}25.2 \pm 0.9 \\
{[14-37]}\end{array}$ & $\begin{array}{c}28.6 \pm 1.2^{\mathrm{A}} \\
{[19-45]}\end{array}$ & $\begin{array}{c}27.1 \pm 1.1^{B} \\
{[15-40]}\end{array}$ & $\begin{array}{c}26.2 \pm 1.3^{c} \\
{[16-42]}\end{array}$ \\
\hline $\operatorname{ALT}(U / L)$ & $\begin{array}{c}28.1 \pm 1.3 \\
{[10-41]}\end{array}$ & $\begin{array}{c}26.6 \pm 1.4 \\
{[10-41]}\end{array}$ & $\begin{array}{c}27.1 \pm 1.6 \\
{[9-47]}\end{array}$ & $\begin{array}{c}32.9 \pm 1.9^{A} \\
{[8-55]}\end{array}$ & $\begin{array}{c}31.5 \pm 1.8^{B} \\
{[8-53]}\end{array}$ & $\begin{array}{c}27.2 \pm 1.8^{c} \\
{[8-47]}\end{array}$ \\
\hline Lymphocytes (cels $\times 10^{3} / \mathrm{mm}^{3}$ ) & $\begin{array}{l}2.36 \pm 0.08 \\
{[1.61-3.46]}\end{array}$ & $\begin{array}{c}2.4 \pm 0.1 \\
{[1.44-4.13]}\end{array}$ & $\begin{array}{c}2.4 \pm 0.1 \\
{[1.64-4.02]}\end{array}$ & $\begin{array}{l}2.1 \pm 0.8 \\
{[0.9-3.1]}\end{array}$ & $\begin{array}{c}2.3 \pm 0.1^{\mathrm{B}} \\
{[1.29-2.67]}\end{array}$ & $\begin{array}{c}2.5 \pm 0.1^{\mathrm{A}} \\
{[1.58-3.12]}\end{array}$ \\
\hline Leukocytes (cells $\times 10^{3} / \mathrm{mm}^{3}$ ) & $\begin{array}{l}7.67 \pm 0.25 \\
{[4.13-9.72]}\end{array}$ & $\begin{array}{l}7.80 \pm 0.22 \\
{[5.06-10.8]}\end{array}$ & $\begin{array}{l}7.64 \pm 0.22 \\
{[4.47-9.87]}\end{array}$ & $\begin{array}{c}6.75 \pm 0.18^{C} \\
{[4.07-9.3]}\end{array}$ & $\begin{array}{c}7.37 \pm 0.19^{B} \\
{[4.37-9.8]}\end{array}$ & $\begin{array}{l}8.77 \pm 0.24^{\mathrm{A}} \\
{[5.8-12.55]}\end{array}$ \\
\hline Platelets (cells $\times 10^{3} / \mathrm{mm}^{3}$ ) & $\begin{array}{c}243.7 \pm 9.8 \\
{[132-376]}\end{array}$ & $\begin{array}{c}237.9 \pm 8.3 \\
{[131-359]}\end{array}$ & $\begin{array}{c}238.7 \pm 8.1 \\
{[121-335]}\end{array}$ & $\begin{array}{c}233.6 \pm 4.6 \\
{[186-291]}\end{array}$ & $\begin{array}{c}235.4 \pm 4.9 \\
{[192-297]}\end{array}$ & $\begin{array}{c}237.6 \pm 4.8 \\
{[181-275]}\end{array}$ \\
\hline Potassium (mEq/L) & $\begin{array}{c}4.35 \pm 0.02 \\
{[4.1-4.6]}\end{array}$ & $\begin{array}{c}4.32 \pm 0.02 \\
{[3.9-4.5]}\end{array}$ & $\begin{array}{c}4.37 \pm 0.03 \\
{[4.0-4.7]}\end{array}$ & $\begin{array}{c}4.1 \pm 0.02 \\
{[3.8-4.3]}\end{array}$ & $\begin{array}{c}4.1 \pm 0.02 \\
{[3.8-4.4]}\end{array}$ & $\begin{array}{l}4.1 \pm 0.1 \\
{[3.8-4.3]}\end{array}$ \\
\hline Sodium (mEq/L) & $\begin{array}{c}139.9 \pm 0.4 \\
{[137-146]}\end{array}$ & $\begin{array}{c}140.1 \pm 0.4 \\
{[136-145]}\end{array}$ & $\begin{array}{c}140.2 \pm 0.3 \\
{[137-144]}\end{array}$ & $\begin{array}{c}139.9 \pm 0.2 \\
{[136-142]}\end{array}$ & $\begin{array}{c}140.7 \pm 0.2 \\
{[138-143]}\end{array}$ & $\begin{array}{c}141.6 \pm 0.4 \\
{[138-149]}\end{array}$ \\
\hline
\end{tabular}

*Values correspond to means \pm standard error of three determinations and minimum and maximum value. Values not sharing similar letter in the same line referent to control group (a) and treatment group ( $A$ ) are different $(p<0.05)$ in Tukey test. 
101.4\%, $\mathrm{p}=0.038$ ). Protein and fiber intake also improved at the end of this period $(82.04 \%$ vs $93.53 \%, \mathrm{p}=0.043$, and $50.44 \%$ vs $63.6 \%, p=0.034$, respectively). Ingestion of calcium ( $\mathrm{p}=0.002)$, ascorbic acid ( $\mathrm{p}=0.035)$, vitamin $\mathrm{E}$ $(\mathrm{p}=0.012)$ and retinol $(\mathrm{p}=0.041)$ increased.

We found similar changes in the supplemented group. Energy adequacy rose from 78.55 to $92.20 \%(\mathrm{p}=0.034)$ in two months. Intake of carbohydrates $(72.61 \%$ vs $91.1 \%$, $\mathrm{p}=0.021)$, lipids $(94.66 \%$ vs $107.2 \%, \mathrm{p}=0.001)$, proteins (79.24\% vs $105.6 \%, \mathrm{p}=0.001)$ and fibers $(49.96 \%$ vs $74.48 \%$, $\mathrm{p}=0.002)$ improved in this group after two months. Calcium and vitamin $E$ ingestion also increased $(\mathrm{p}=0.002$ and 0.014, respectively).

Table 1 shows body weight, BMI, fat and lean body mass in the control and supplemented groups. There was a significant reduction of body weight and BMI in the control group ( $\mathrm{p}=0.029$ and 0.003 , respectively), whereas in the supplemented group we found increased BMI $(\mathrm{p}=0.041)$. Differences between groups did not reach statistical significance. Table 1 also shows TSF, BSF, SISF and SESF skinfolds in both groups.

In the control group only, we found decreased MAMC and AMA in the follow-up ( $\mathrm{p}=0.003 \mathrm{e} \mathrm{p}=0.019)$, whereas AFA increased ( $\mathrm{p}=0.042)$. MAC and wrist circumference did not change in the supplemented patients $(\mathrm{p}=0.062 \mathrm{e}$ $\mathrm{p}=0.090$, respectively) (Table 1$)$.

There was significant improvement in serum albumin levels $(\mathrm{p}=0.001)$, total lymphocyte count $(\mathrm{p}=0.004)$ and white blood cell count (0.002) among supplemented patients. In this group, CK, AST and ALT levels significantly decreased ( $\mathrm{p}=0.028,0.004$ and 0.003 , respectively), as depicted on Table 2.

ALSFRS-R scores in both groups are on Table 3.

\section{DISCUSSION}

Patients with ALS included in this study share the characteristic epidemiologic features of the disease $\mathrm{e}^{23}$. We did not find relevant differences between patients with bulbar and appendicular predominance.

Most patients had low income $(88 \%$ received $<\$ 1,000.00)$ and education level (80\% studied up to the $4^{\text {th }}$ grade). Ten patients (62\%) had 5 or 6 daily meals, four (25\%) had 4, and two (13\%) had 3 daily meals.

Rio and Cawadias ${ }^{24}$ reviewed nutritional guidelines for patients with ALS in the United Kingdom and found that calorie and protein supplementation was the cornerstone of nutritional therapy. Heffernan et al. ${ }^{25}$ also emphasized the relevance of education and nutritional intervention in these individuals. In a previous study with 20 patients, authors were able to modify the nutritional status classification in $70 \%$ of individuals after oral supplementation. They have shown that oral supplementation avoided worsening the nutritional status, but could not normalize the overall nutritional adequacy ${ }^{26}$. In accordance with Karsarskis et al. ${ }^{27}$ and Desport et al. ${ }^{1}$, we have found progressive decline of body weight and BMI in non-supplemented patients. However, BMI improved in patients that received oral supplementation. In this group, fatty and lean body mass did not decline in the follow-up, leading to higher PCMS.

Skinfolds are a useful clinical method to evaluate body composition, namely the proportion of fat mass ${ }^{28,29}$. We found in the control group that BSF and SESF increased in the follow-up, thus suggesting higher proportions of body fat mass. Increased TSF in supplemented patients may have been caused by higher food intake during the study. Slowie ${ }^{4}$ identified a relationship between reduced food intake and smaller TSF. Modifications in TSF may have contributed to improved PCMS scores in this group.

MAMC and AMA are markers of striated muscle protein mass $^{28}$. MAC and wrist circumference did not change in the follow-up in either group (Table 1). MAMC and AMA declined in the control group, whereas AFA increased; this may indicate a fatty change in the skeletal muscle due to chronic denervation. MAMC and AMA remained stable in the supplemented group, but AFA declined, suggesting preserved lean body mass and reduced fat body mass. Similar findings were reported by Stanich et $\mathrm{al}^{26}{ }^{26}$ among patients with ALS receiving hypercaloric supplementation. Karsarsis et al. ${ }^{27}$ identified progressive AMA decline over disease course.

Patients in both groups were classified as eutrophic according to the BMI. However, PCMS takes into account body composition measures and is therefore a more reliable tool to evaluate nutritional status. In the control group, PCMS were 80.13, 77.67 and 79.93 at the onset and after 2 months and 4 months of follow-up, respectively. Meanwhile, PCMS progressively increased among the supplemented patients (75.26, 77.13 and 78.89). Despite the different courses, patients in both groups were classified as having moderate malnutrition (70-80\%).

Table 3. ALSFRS-R: Level of significance among the first and second time of control and supplemented groups.

\begin{tabular}{|c|c|c|c|c|c|c|}
\hline & \multicolumn{3}{|c|}{ Control group* } & \multicolumn{3}{|c|}{ Treatment group* } \\
\hline & 0 & 4 & p-value & 0 & 4 & $\mathrm{p}$-value \\
\hline ALSFRS-R & $27.0 \pm 3.8^{\mathrm{a}}$ & $23.6 \pm 4.4^{b}$ & 0.03 & $29.1 \pm 2.4$ & $27.0 \pm 3.5$ & 0.17 \\
\hline
\end{tabular}

ALSFRS-R: score of Amyotrophic Lateral Sclerosis Functional Rating Scale-Revised at first (0) and second times (4 months), respectively. Values correspond to means \pm standard error of three determinations. Values not sharing similar letters in the same line are different $(p<0.05)$ by the Tukey test. 
Clinical and biochemical parameters showed normal values for albumin, pre-albumin and C-reactive protein (CRP) in patients with ALS. Kasarskis et al. ${ }^{27}$ previously found that pre-albumin and retinol-binding protein serum levels did not change over disease course. These results are much similar to the ones observed in our supplemented group. Desport et al. ${ }^{1}$ also found normal values for CRP, white blood cell and total lymphocyte counts in ALS. We found increased serum albumin, white blood cell and total lymphocyte counts in supplemented patients; this may be related to immunomodulatory properties of milk proteins ${ }^{28}$. Muscle turnover, expressed by CK, AST and ALT levels, also decreased in this group.

The bioactive properties of milk whey protein were observed in studies made in order to analyze the different effects of food proteins in the immune system of animals. It was noticed that the whey protein isolate contains high levels of glutathione, and its precursors cystine and glutamylcystine, and that the combination of these factors plays an important role in maintaining the necessary cellular glutathione levels to guarantee an efficient immune response ${ }^{29}$. Considering these bioactive properties, benefic effects may be implied in the analyzed parameters. Different from proteins, modified starch used in the treatment group was only composed by carbohydrates, calculated as part of the recommended daily energetic ingestion of the individuals' diet.

ALSFRS-R is a reliable tool useful to predict survival in $\mathrm{ALS}^{30,31}$, survival after mechanical non-invasive ventilation, and to estimate overall functioning of patients with ALS $^{32}$. ALSFRS-R scores did not change among supplemented patients $(\mathrm{p}=0.173)$, but significantly declined in the control group ( $\mathrm{p}=0.003$ ).

In Conclusion, during the follow-up study, intake of carbohydrates, lipids, proteins, fibers, calcium and vitamin E significantly increased in both control and supplemented groups. BMI, MAMC and AMA declined over time in the control group, which received maltodextrin. In the supplemented group, however, serum albumin, white blood cell and total lymphocyte counts increased. Lean body mass seems to be preserved in this group, and skeletal muscle turnover markers (CK, AST and ALT) declined. The ALSFRS-R scores did not change in the supplemented group, while patients in the control group presented a progressive decline. Our results indicate that 70\%WPI:30\%MS improved the nutritional status of patients with ALS, and may be useful in the clinical management of the disease.

\section{REFERENCES}

1. Desport JC, Preux PM, Magy L, et al. Factor correlated with hypermetabolism in patients with amyotrophic lateral sclerosis. Am J Clin Nutr 2001;74:328-334

2. Strand EA, Miller RM, Yorkston KM, Hillel AD. Management of oral-pharyngeal dysphagia symptoms in amyotrophic lateral sclerosis. Dysphagia 1996; 11:129-139.
3. Desport JC, Preux PM, Truong TC, Vallat JM, Sautereau D. Couratier P. Nutritional status is a prognostics factor for survival in ALS patients. Neurology 1999:53:1059-1063.

4. Slowie LA, Paige MS, Antel JP. Nutritional considerations in the management of patients with ALS amyotrophic lateral sclerosis. J Am Diet Assoc 1983;83: 44-47.

5. Wright $L$, Cotter D, Hickson M, Frost. Comparison of energy and protein intakes of older people consuming a texture modified diet with normal hospital diets. J Human Nutr Diet 2005;18:213-219.

6. Silva LBC, Mourão LF, Silva AA, et al. Avaliação da ingestão alimentar de indivíduos com esclerose lateral amiotrófica. Rev Bras Nutr Clin 2008:23:5-12.

7. ADA: National Dysphagia Diet: Standardization for Optimal Care. National Dysphagia Diet Task Force. Chicago: The American Dietetics Association, 2003:50.

8. World Federation of Neurology Research Group on Neuromuscular Diseases. El Escorial revisited: revised criteria for the diagnosis of amyotrophic lateral sclerosis. HYPERLINK http:// www.wfnals.org/articles/elescorial1998.htm

9. Fisberg RM, Martini LA, Slater B. Métodos de Inquéritos Alimentares. In: Fisberg RM, Slater B, Marchioni DML, Martini LA (Eds). Inquéritos Alimentares: Métodos e bases científicos. São Paulo: Manole, 2005:2-7.

10. Monteiro JBR, Esteves EA. Diet Pro versão 4.0. Sistema de suporte à avaliação nutricional e prescrição de dietas [CD-ROM]. Viçosa:Agromídia Software; 2001.

11. Tabela Brasileira de Composição de Alimentos. NEPA-UNICAMP-Versão II-2th ed. Campinas:NEPA-UNICAMP;2006:42.

12. U.S.Departament of Agriculture. Composition of Foods. Raw, Processed, Prepared USDA National Nutrient Database for Standard Reference. Release 16. Nutrient Data Laboratory homepage. HYPERLINK http://www.nal.usda.gov/ fnic/foodcomp/Data/SR18/SR18doc.pdf.

13. National Academic of Sciences. National Research Council. Recommended dietary allowances. 10th ed. Washington: National Academic Press, 1989:115.

14. Vannucchi H, Menezes EW, Campana AO, Lajolo FM. Aplicações das recomendações adaptadas à população brasileira. Ribeirão Preto: Legis Suma, 1990;156.

15. Long CL, Schaffel N, Geiger JW. Metabolic response to injury and illness: Estimation of energy and protein needs from indirect calorimetry and nitrogen balance. JPEN 1979;3:452-456

16. Grant JP, Custer PB, Thurlon J. Current techniques of nutrition assessment. In Handbook of total parenteral nutrition. Philadelphia: Saunders, 1980:12-23.

17. Chumlea WC, Roche AF, Steinbaugh ML. Estimating stature from knee height for persons 60 to 90 years of age. J Am Geriatric Soc 1985;33:116-120.

18. Lohman TG, Roche AF, Martorell R. Anthropometric standardization reference manual. Champaign: Human Kinetics, 1989:39-54.

19. Heymsfield SB, McManus S, Smith J, Stevens V, Nixon DW. Anthropometric measurement of muscle mass: revised equations for calculating bone-free arm muscle area. Am J Clin Nutr 1982;36:680-690.

20. Frisancho AR. New norms of upper limb fat and muscle areas for assessment of nutritional status. Am J Clin Nutr 1981;34:540-545

21. DeHoog S. Avaliação do Estado Nutricional. In: Mahan LK, Stump SE. (Eds). Alimentos, nutrição e dietoterapia. 9 Ed. São Paulo:Roca, 1998:371-395.

22. Cedarbaum JM Stambler N. Malta E. Fuller C. Hilt D. Thurmond B. The ALS FRS-R: a revised ALS functional rating scale that incorporates assessments of respiratory function. J Neurol Sci 1999;169:13-21.

23. Dietrich-Neto F, Callegaro D, Dias-Tosta E, et al. Amyotrophic lateral sclerosis in Brazil: 1998 national survey. Arq Neuropsiquiatr 2000;58:607-615.

24. Rio A, Cawadias E. Nutritional advice and treatment by dietitians to patients with amyotrophic latera sclerosis/motor neurone disease: a survey of current practice in England, Wales, Northern Ireland and Canada. J Hum Nutr Diet 2007;20:1-13.

25. Heffernan C, Jenkinson C, Holmes T, et al. Nutritional management in MND/ ALS patients: an evidence based review. Amyotroph Lateral Scler Other Motor Neuron Disord 2004;5:72-83.

26. Stanich P, Pereira AML, Chiappeta ALML, Nunes M, Oliveira ASB, Gabbai AA. Suplementação nutricional em pacientes com doença do neurônio motor/ esclerose lateral amiotrófica. Rev Bras de Nutr Clin 2004;19:70-78.

27. Kasarskis E, Berryman S, Vanderleest JG, Schneider AR, McClain CJ. Nutritional status of patients with amyotrophic lateral sclerosis: relation to the proximity of death. Am J Clin Nutr 1996:63:130-137.

28. Mercier A, Gauthier SF, Fliss I. Immunomodulating effects of whey proteins and their enzymatic digests. Int Dairy J 2004;14:175-183.

29. Bounous G, Molson JH. The antioxidant system. Anticancer Res 2003;23:14111416.

30. Silva LBC, Mourão LF, Silva AA, et al. Amyotrophic lateral sclerosis: combined nutritional, respiratory and functional assessment. Arq Neuropsiquiatr 2008:66:354-359.

31. Gordon PH, Cheng B, Montes J, Doorish A, Albert SM, Mitsumoto H. Outcome measures for early phase clinical trials in amyotrophic lateral sclerosis. Amyotroph Lateral Scler Other Motor Neuron Disord 2007;27:1-4.

32. Lo Coco D, Marchese S, La Bella V, Piccoli T, Lo Coco A. The amyotrophic lateral sclerosis functional rating scale predicts survival time in amyotrophic lateral sclerosis patients on invasive mechanical ventilation. Chest 2007;132:64-69. 